\title{
Effect of Vitamin D Supplementation on Glycemic Control in Prediabetes: A Meta-Analysis
}

\author{
Yujing Zhang, Yuan Xue, Dongdong Zhang, Yaping Liu, Ze Xu, Jiaojiao Gao, Wenjie Li and Xing Li * \\ College of Public Health, Zhengzhou University, Zhenghzou 450000, China; 15093458874@163.com (Y.Z.); \\ xueyuansnow@163.com (Y.X.); dongdongzhang23@163.com (D.Z.); liuyaping_tang@163.com (Y.L.); \\ ze1995@163.com (Z.X.); gjjatl@163.com (J.G.); lwj@zzu.edu.cn (W.L.) \\ * Correspondence: lixing530@zzu.edu.cn
}

Citation: Zhang, Y.; Xue, Y.; Zhang, D.; Liu, Y.; Xu, Z.; Gao, J.; Li, W.; Li, X. Effect of Vitamin D Supplementation on Glycemic Control in Prediabetes: A Meta-Analysis. Nutrients 2021, 13, 4464. https://doi.org/10.3390/ nu13124464

Academic Editors: Giovanni Passeri and Sandro Giannini

Received: 10 November 2021 Accepted: 9 December 2021 Published: 14 December 2021

Publisher's Note: MDPI stays neutral with regard to jurisdictional claims in published maps and institutional affiliations.

Copyright: (c) 2021 by the authors. Licensee MDPI, Basel, Switzerland. This article is an open access article distributed under the terms and conditions of the Creative Commons Attribution (CC BY) license (https:// creativecommons.org/licenses/by/ $4.0 /)$.

\begin{abstract}
Clinical research results of vitamin D supplementation in the improvement of prediabetes remain controversial. Accordingly, a literature search was conducted of PubMed, Embase (Ovid), and Web of Science prior to 9 November 2021. Randomized controlled studies reported that the following indicators were included: body mass index (BMI), fasting blood glucose (FBG), $2 \mathrm{~h}$ oral glucose tolerance test plasma glucose (2h-PG), hemoglobin $\mathrm{A} 1 \mathrm{c}(\mathrm{HbA1c})$, insulin resistance by homeostasis model assessment (HOMA-IR), homeostasis model assessment of $\beta$-cell function (HOMA-B), and fasting insulin (FINS). Twenty-nine articles $(N=3792)$ were included in the present meta-analysis. Intriguingly, vitamin D supplementation resulted in a vast improvement in FBG (standardized mean difference $(\mathrm{SMD})=-0.38 ; 95 \% \mathrm{CI}:-0.59,-0.16), \mathrm{HbA} 1 \mathrm{c}(\mathrm{SMD}=-0.14 ; 95 \% \mathrm{CI}:-0.22,-0.06)$ and FINS (SMD $=0.18 ; 95 \% \mathrm{CI}:-0.26,-0.09)$, but not in other outcomes. However, preferred changes were observed in subgroups, as follows: Asia $\left(\mathrm{SMD}_{2 \mathrm{~h}-\mathrm{PG}}=-0.25,95 \% \mathrm{CI}:-0.45,-0.04\right)$, study duration $\geq 1$ year $\left(\mathrm{SMD}_{\text {HOMA-IR }}=-0.44,95 \% \mathrm{CI}:-0.81,-0.06\right)\left(\mathrm{SMD}_{\text {HOMA-B }}=0.34,95 \% \mathrm{CI}\right.$ : 0.01, 0.66), baseline $25(\mathrm{OH}) \mathrm{D}<50 \mathrm{nmol} / \mathrm{L}\left(\mathrm{SMD}_{2 \mathrm{~h}-\mathrm{PG}}=-0.23,95 \% \mathrm{CI}:-0.39,-0.06\right)$, and baseline $25(\mathrm{OH}) \mathrm{D} \geq 50 \mathrm{nmol} / \mathrm{L}$ $\left(\mathrm{SMD}_{\text {HOMA-IR }}=-0.50,95 \% \mathrm{CI}:-0.96,-0.03\right)$. In conclusion, oral supplementation of vitamin $\mathrm{D}$ has shown better effects in improving FBG, $\mathrm{HbA1c}$, and FINS compared with controls among prediabetics; long-term vitamin D supplementation could have additional effects in participants with vitamin $D$ deficiency for 2h-PG, HOMA-IR, and HOMA-B.
\end{abstract}

Keywords: vitamin D; prediabetes; glycemic control; meta-analysis

\section{Introduction}

Prediabetes is defined as impaired fasting glucose (IFG) and/or impaired glucose tolerance (IGT), which is characterized by hyperglycemia, insulin resistance, and $\beta$-cell dysfunction. It is estimated that the proportion of global IGT in 2019 was $7.5 \%$ (374 million) by the International Diabetes Federation (IDF), and $8.6 \%$ of adults (548 million) will suffer from IGT by 2045 [1]. Prediabetes is a reversible stage; effective early intervention, such as diet control, exercise enhancement, and drug intervention, can reduce the risk of type 2 diabetes mellitus (T2DM) by $40-70 \%$. Without these interventions, 5-10\% of the prediabetic population will develop diabetes every year, and up to $70 \%$ will ultimately develop diabetes [2]. Therefore, it is vital to prevent or delay the progression of prediabetes to diabetes.

Vitamin D (VD) is a fat-soluble vitamin that performs its biological functions in the form of vitamin $\mathrm{D}_{2}$ and vitamin $\mathrm{D}_{3}$. Epidemiological studies have shown that patients with T2DM and prediabetes have lower serum 25(OH) $\mathrm{D}_{3}$ levels [3,4]. A four-year followup study suggested that low levels of serum $25(\mathrm{OH}) \mathrm{D}$ might be linked to the increased incidence of prediabetes or T2DM in Chinese individuals [5]. Two meta-analyses observed that the administration of vitamin D led to a reduction in glucose and hemoglobin A1c $(\mathrm{HbA} 1 \mathrm{c})$, regardless of intramuscular injection and oral supplementation [6,7]. Therefore, 
more randomized controlled trials (RCTs) are needed to explore the association between vitamin D supplementation and prediabetes.

The aim of the current meta-analysis is to assess the effect of oral vitamin D supplementation on glycemic control in the prediabetic population.

\section{Materials and Methods}

This study was conducted on the basis of the Preferred Reporting Items for Systematic Reviews and Meta-Analysis (PRISMA) statement guidelines.

\subsection{Literature Search}

Articles were searched in the electronic databases of PubMed, Embase (Ovid), and Web of Science from inception to 9 November 2021 using the following search terms: ("vitamin D" OR "vitamin $\mathrm{D}_{2}$ " OR "vitamin $\mathrm{D}_{3}$ " OR "alphacalcidol" OR "alfacalcidol" $\mathrm{OR}$ "cholecalciferol" OR "ergocalciferol" OR "paricalcitol" OR "doxercalciferol" OR "calcitriol" OR "25-hydroxyvitamin D" OR "25(OH)D") AND ("prediabetes" OR "pre-diabetes" OR "prediabetic" OR "pre-diabetic" OR "impaired glucose tolerance" OR "impaired fasting glucose" OR "impaired plasma glucose" OR "insulin resistance" OR "hyperglycemia").

\subsection{Selection of Studies}

The articles were screened and assessed according to our inclusion and exclusion criteria, by two authors independently.

Inclusion criteria:

(1) Population: adults ( $\geq 18$ years) with prediabetes. Prediabetes criteria [8,9]: (1) IFG: fasting blood glucose (FBG) 6.1-6.9 mmol/L (World Health Organization (WHO)) or 5.6-6.9 mmol/L (American Diabetes Association (ADA)); or (2) IGT: 2h oral glucose tolerance test plasma glucose (2h-PG) 7.8-11.0 mmol/L during an oral glucose tolerance test; or (3) hemoglobin A1c (HbA1c) 5.7-6.4\% (ADA).

(2) Intervention: treatment with vitamin D or analogue supplements orally, regardless of the types, dosages, durations, or routines, either with or without calcium (Ca).

(3) Comparison intervention: placebo or nothing.

(4) RCT design.

(5) Eligible studies must have reported at least one of the following coprimary outcomes of interest as defined by the investigators: body mass index (BMI), FBG, 2h-PG, HbA1c, insulin resistance by homeostasis model assessment (HOMA-IR), homeostasis model assessment of $\beta$-cell function (HOMA-B), and fasting insulin (FINS). Available sample size, mean and standard deviation (SD) of relevant indexes or data were provided so that mean and SD could be calculated.

(6) Written in English or Chinese.

Exclusion Criteria:

(1) Animal studies, case reports, reviews, or abstracts.

(2) Repeated or overlapped publication.

(3) Trials of participants with diabetes.

(4) Duplicated publications; only the first publication reporting related outcomes was included.

\subsection{Data Extraction and Risk of Bias Assessment}

The following details of each included trial were extracted: last name of the first author, year of publication, country or region, participants, sample size, mean age of the participants, vitamin D type and dose, control group, duration of study, outcome measures.

Two reviewers independently assessed the risk of trial bias based on Cochrane's risk of bias assessment tool and designated the following items as "high" or "low" or "unclear": random sequence generation; allocation concealment; blinding of participants and personnel; blinding of outcome assessment; selective reporting; and other sources of bias [10]. 


\subsection{Statistical Analysis}

An inquiry to authors was set up when SDs were not reported and could not be calculated from the available data. In the absence of data from authors, the SD was estimated from SDs reported by other studies [7].

The effect of vitamin D supplementation on glycemic control was evaluated in the prediabetic population using the standardized mean difference (SMD) and $95 \%$ confidence interval (CI). The forest plot was used to visually assess pooled estimates, and it was also used to identify heterogeneity by $\mathrm{I}^{2}$ and $p$ value [11]; $p \leq 0.05$ and $\mathrm{I}^{2}>50 \%$ was considered as heterogeneity. The random-effect model was used to aggregate the results; otherwise, the fixed-effect model was used. To explore the sources of high heterogeneity, subgroup analysis and meta-regression were performed, and sensitivity analysis was used to assess the stability of the results. Subgroup analyses were conducted to illustrate the impacts of certain characteristics: continent (Asia, Europe, and America); vitamin D supplementation (VD vs. placebo, VD + Ca/omage-3 vs. Ca/omage-3, VD+Ca vs. placebo, VD vs. nothing); study duration $(\geq 1$ year, $<1$ year); baseline $25(\mathrm{OH}) \mathrm{D}(<50 \mathrm{nmol} / \mathrm{L}$, $\geq 50 \mathrm{nmol} / \mathrm{L}$ ); biospecimen (serum, plasma, unclear) and HOMA-IR and HOMA-B for different calculating methods (type 1, type 2). The publication bias was defined as "the publication or non-publication of research findings, depending on the nature and direction of the results" in accordance with the Cochrane Handbook, and it was assessed by Begg's test and funnel plots. Meta-regression was performed to detect published year, continent, vitamin D supplementation, study duration, and baseline $25(\mathrm{OH}) \mathrm{D}$. All the preceding analyses were conducted using Stata 12.0 (Stata, College Station, TX, USA) and Cochrane Review Manager (RevMan) 5.4. The value of $p<0.05$ was considered statistically significant.

\section{Results}

\subsection{Literature Search}

A total of 12,741 potential articles were included after a preliminary search of PubMed, Embase (Ovid), and Web of Science. Among the retrieved articles, 8654 articles were left after removing duplicates. After screening the titles and abstracts, 8589 articles were excluded owing to an irrelevance of the topic. After assessing the full text of the remaining 65 articles, 36 articles were excluded for the following reasons: (1) meeting abstracts $(n=17)$, (2) no control group $(n=3)$, (3) no relevant indexes $(n=7)$, (4) intervention individuals were not or not only prediabetics $(n=3)$, (5) duplicated publications $(n=2)$, (6) protocols $(n=2),(7)$ not in English or Chinese $(n=1)$, and (8) vitamin D intramuscular injection $(n=1)$. A total of 29 articles $(N=3792)$ were included in this meta-analysis (BMI: $n=23$; FBG: $n=25$; 2h-PG: $n=18$; HbA1c: $n=20$; HOMA-IR: $n=21$; HOMA-B: $n=10$; FINS: $n=15)$. The process of screening and selection is shown in Figure 1.

\subsection{Study Characteristics}

Table 1 showed the characteristics of the 29 selected studies in the meta-analysis. All included studies were published between 2007 and 2020 and were carried out in three continents: 12 studies were performed in Asia, 10 studies were conducted in Europe, and seven studies in America. The numbers of participants ranged from 29 to 484, with the mean age ranging from 40 to 76 years old. The intervention periods of vitamin $D$ supplementation were from 8 weeks to 7 years. Twenty-four studies used vitamin $D_{3}$ as the intervention agent, and vitamin $\mathrm{D}_{2}$ was used in only one study; moreover, two studies included both vitamin $\mathrm{D}_{3}$ and vitamin $\mathrm{D}_{2}$ interventions, while the remaining two studies failed to describe the specific type of vitamin $\mathrm{D}$. There were 18 studies that applied vitamin $\mathrm{D}$ intervention alone; 10 studies provided vitamin $\mathrm{D}$ and calcium co-supplementation (one study involved two intervention groups: vitamin $\mathrm{D}_{3}$ and calcium/calcium-placebo co-supplementation); and one study involved vitamin $\mathrm{D}_{3}$ and omega- 3 co-supplementation or vitamin $\mathrm{D}_{3}$ supplementation alone. 


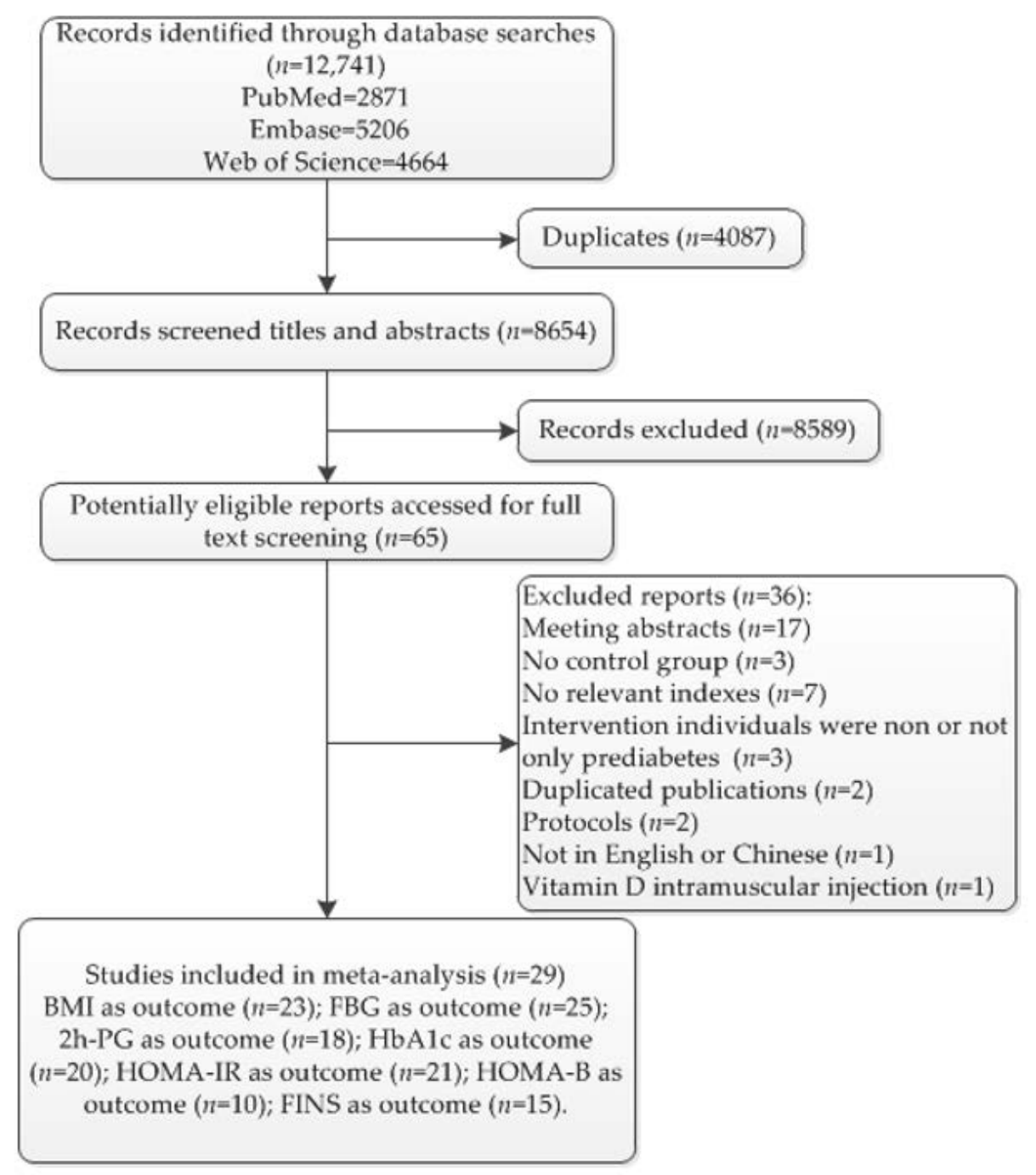

Figure 1. PRISMA flow diagram of the selected studies, indicating the used exclusion criteria. Abbreviations: BMI: body mass index; FBG: fasting blood glucose; 2h-PG: $2 \mathrm{~h}$ oral glucose tolerance test plasma glucose; HbA1c: glycosylated hemoglobin A1c; HOMA-IR: insulin resistance by homeostasis model assessment; HOMA-B: homeostasis model assessment of $\beta$-cell function; FINS: fasting insulin.

\subsection{Risk of Bias}

Figure S1A shows the comprehensive details of the risk of bias assessment. The principal problem detected in the 29 RCTs was that there exists a high risk of bias due to (1) allocation concealment, (2) binding of participants and personnel, and (3) incomplete outcome data. Figure S1B summarizes the proportion of trials with low, unclear, and high risks of bias for each domain. Selection and attrition bias were the primary sources of bias.

\subsection{Main Analysis}

\subsubsection{BMI}

The change in BMI after vitamin D intervention was reported in 23 studies $(N=2854)$ $[12,14,17,18,20-23,25-37,39,40]$. Random-effects meta-analysis showed no difference in BMI changes between vitamin D supplementation and controls (SMD $=0.01 ; 95 \% \mathrm{CI}:-0.22$, $0.24 ; p=0.935$ ) (Figure S2), while the results showed statistically significant heterogeneity $\left(\mathrm{I}^{2}=88.7 \%, p<0.001\right)$. Furthermore, subgroup analyses for continent, vitamin D supplementation, baseline $25(\mathrm{OH}) \mathrm{D}$, and duration of the studies were also conducted; the details are presented in Table 2. Only vitamin D + Ca vs. the placebo subgroup exhibited a significant decrease, but conclusions could not be drawn because it only contained one study. 
Table 1. Characteristics of studies included in the meta-analysis.

\begin{tabular}{|c|c|c|c|c|c|c|c|c|c|}
\hline Study & Year & Country & Participants & $N(\mathrm{I} / \mathrm{C})$ & Age (Years) & Intervention & Control & Duration Period & Outcomes \\
\hline Pittas et al. [12] & 2007 & USA & IFG & $45 / 47$ & $\begin{aligned} \text { I: } 71.1 & \pm 0.7 \text { C: } 71.3 \\
& \pm 0.8\end{aligned}$ & $\mathrm{VD}_{3} 700 \mathrm{IU} /$ day + Ca $500 \mathrm{mg} /$ day & placebo & 3 years & (1) (2) (5) \\
\hline de Boer et al. & 2008 & USA & IFG & $172 / 178$ & NA & $\mathrm{VD}_{3} 400 \mathrm{IU} /$ day $+\mathrm{Ca} 1000 \mathrm{mg} /$ day & placebo & 7 years & (2) (5) (7) \\
\hline Jorde et al. [14] & 2010 & Norway & Prediabetes & $34 / 31$ & NA & $\begin{array}{c}\mathrm{VD}_{3} 20,000 \text { or } 40,000 \mathrm{IU} / \text { week + Ca } 500 \\
\text { mg/day }\end{array}$ & $\begin{array}{l}\text { placebo }+ \text { Ca } 500 \\
\text { mg/day }\end{array}$ & 1 year & (1) (2) (3) (4) (5) (7) \\
\hline Mitri et al. [15] & 2011 & USA & Prediabetes & $46 / 46$ & $\begin{array}{l}\text { I: } 57 \pm 9.59 \text { C: } 58 \pm \\
\quad 9.60\end{array}$ & $\begin{array}{c}\mathrm{VD}_{3} 2000 \mathrm{IU} / \text { day }+ \text { Ca } 800 \mathrm{mg} / \text { day or } \\
\text { Ca-placebo }\end{array}$ & $\begin{array}{l}\text { VD-placebo + Ca } 800 \\
\text { mg/day or } \\
\text { Ca-placebo }\end{array}$ & 16 weeks & (2) (3) (4) \\
\hline Harris et al. [16] & 2012 & USA & Prediabetes & $43 / 46$ & $\begin{aligned} \text { I: } 71.1 & \pm 0.7 \text { C: } 56.3 \\
& \pm 12.3\end{aligned}$ & $\mathrm{VD}_{3} 4000 \mathrm{IU} /$ day $+\mathrm{Ca} 600 \mathrm{mg} /$ day & $\begin{array}{l}\text { placebo + Ca } 600 \\
\text { mg/day }\end{array}$ & 12 weeks & (2) (3) (4) (5) (7) \\
\hline $\begin{array}{l}\text { Davidson et al. } \\
\quad[18]\end{array}$ & 2013 & USA & Prediabetes & $56 / 53$ & NA & $\begin{array}{c}\mathrm{VD}_{3} 88,865 \mathrm{IU} / \text { week (range } 64,731-13,4446 \\
\text { IU/week) }\end{array}$ & placebo & 1 year & (1) (2) (3) (4) (5) (6) \\
\hline $\begin{array}{l}\text { Hoseini et al. } \\
{[19]}\end{array}$ & 2013 & Iran & Prediabetes & $21 / 15$ & I: $\begin{aligned} 46.3 & \pm 6.5 \text { C: } 48.9 \\
& \pm 6.1\end{aligned}$ & $\begin{array}{l}\text { VD 50,000 IU/week or every other week + } \\
\text { Ca } 500 \mathrm{mg} / \text { day }\end{array}$ & Ca 500 mg/day & 12 weeks & (2) (4) (5) (6) \\
\hline Sollid et al. [20] & 2014 & Norway & Prediabetes & $242 / 242$ & NA & $\mathrm{VD}_{3} 20,000 \mathrm{IU} /$ week & placebo & $\begin{array}{l}1 \text { years } \\
\end{array}$ & (1)(2) (3) (4)(5) (7) \\
\hline Dutta et al. [21] & 2014 & India & Prediabetes & $55 / 49$ & NA & $\begin{array}{l}\mathrm{VD}_{3} 60,000 \mathrm{IU} / \text { week for } 8 \text { weeks, then } \\
60,000 \mathrm{IU} / \mathrm{month}+\text { Ca } 500 \mathrm{mg} / \text { day }\end{array}$ & Ca $500 \mathrm{mg} /$ day & $\begin{array}{c}\text { I: } 28.2 \pm 8.83 \text { months } \\
\text { C: } 29.15 \pm 7.69 \\
\text { months }\end{array}$ & (1) (2) (3) (4) (5) (6) \\
\hline $\begin{array}{l}\text { Oosterwerff } \\
\text { et al. [22] }\end{array}$ & 2014 & Netherlands & Prediabetes & $65 / 65$ & NA & $\mathrm{VD}_{3} 1200 \mathrm{IU} /$ day $+\mathrm{Ca} 500$ & $\begin{array}{c}\text { placebo + Ca } 500 \\
\text { mg/day }\end{array}$ & 16 months & (1) (5) \\
\hline $\begin{array}{l}\text { Kuchay et al. } \\
{[23]}\end{array}$ & 2015 & India & Prediabetes & $56 / 55$ & NA & $\begin{array}{c}\mathrm{mg} / \text { day } \\
\mathrm{VD}_{3} 60,000 \mathrm{IU} / \text { week for } 4 \text { weeks and then } \\
60,000 \mathrm{IU} / \mathrm{month}\end{array}$ & nothing & 1 year & (1) (2) (3) (4) \\
\hline $\begin{array}{l}\text { Barengolts et al. } \\
\text { [24] }\end{array}$ & 2015 & USA & Prediabetes & $87 / 86$ & $\begin{aligned} \text { I: } 58.2 & \pm 6.0 \text { C: } 59.8 \\
& \pm 6.0\end{aligned}$ & $\mathrm{VD}_{2} 50,000 \mathrm{IU} /$ week & placebo & 1 year & (4) \\
\hline $\begin{array}{l}\text { Didriksen et al. } \\
{[25]}\end{array}$ & 2015 & Norway & IFG & $18 / 11$ & I: $\begin{aligned} 60.8 & \pm 9.33 \text { C: } 62.0 \\
& \pm 9.41\end{aligned}$ & $\mathrm{VD}_{3} 20,000 \mathrm{IU} /$ week & placebo & $3-5$ years & (1) (4) \\
\hline $\begin{array}{l}\text { Tuomainen et al. } \\
{[26]}\end{array}$ & 2015 & Finland & Prediabetes & $45 / 21$ & NA & $\mathrm{VD}_{3} 1600 / 3200 \mathrm{IU} /$ day & placebo & 5 months & (1) (2) (3) (4) (5) (6) (7) \\
\hline $\begin{array}{l}\text { Nimitphong } \\
\text { et al. [27] }\end{array}$ & 2015 & Thailand & Prediabetes & $29 / 18$ & I: $\begin{aligned} 61.8 & \pm 9.7 \text { C: } 57.9 \\
& \pm 13.3\end{aligned}$ & $\begin{array}{l}\mathrm{VD}_{2} 20,000 \mathrm{IU} / \text { week or } \mathrm{VD}_{3} 15,000 \\
\text { IU/week }\end{array}$ & nothing & 3 months & (1) (2) (3) (4) (5) (6) \\
\hline $\begin{array}{l}\text { Forouhi et al. } \\
{[28]}\end{array}$ & 2016 & UK & Prediabetes & $210 / 111$ & NA & $\begin{array}{l}\mathrm{VD}_{2} 100,000 \mathrm{IU} / \text { month or } \mathrm{VD}_{3} 100,000 \\
\mathrm{IU} / \text { month }\end{array}$ & placebo & 4 months & (1) (4) \\
\hline Jorde et al. [29] & 2016 & Norway & Prediabetes & $116 / 111$ & NA & $\mathrm{VD}_{3} 20,000 \mathrm{IU} /$ week & placebo & 5 years & (1)(2) (3) (4)(5) (7) \\
\hline $\begin{array}{l}\text { Wagner et al. } \\
{[30]}\end{array}$ & 2016 & Sweden & Prediabetes & $21 / 22$ & $\begin{array}{l}\mathrm{I}: 66.52 \pm 4.29 \mathrm{C}: \\
\quad 66.71 \pm 3.01\end{array}$ & $\mathrm{VD}_{3} 30,000 \mathrm{IU} /$ week & placebo & 8 weeks & (1) (2) (3) (4) \\
\hline $\begin{array}{l}\text { Moreira-Lucas } \\
\text { et al. [31] }\end{array}$ & 2017 & Canada & IFG & $35 / 36$ & I: $\begin{aligned} 49.1 & \pm 13.9 \text { C: } 45.6 \\
& \pm 14.3\end{aligned}$ & $\mathrm{VD}_{3} 28,000 \mathrm{IU} /$ week & placebo & 24 weeks & (1) (2) (3) (4) (6) (7) \\
\hline Zarrin et al. [32] & 2017 & Iran & Prediabetes & $51 / 53$ & $\begin{array}{l}\text { I: } 48.11 \pm 7.6 \mathrm{C}: \\
\quad 48.43 \pm 7.7\end{array}$ & $\mathrm{VD}_{3} 1000 \mathrm{IU} /$ day & placebo & 3 months & (1) (2) (4) (5) (7) \\
\hline $\begin{array}{l}\text { Niroomand } \\
\text { et al. [33] }\end{array}$ & 2019 & Iran & Prediabetes & $43 / 40$ & NA & $\begin{array}{l}\mathrm{VD}_{3} 50,000 \mathrm{IU} / \text { week for } 3 \text { months and then } \\
50,000 \mathrm{IU} / \text { month }\end{array}$ & placebo & 6 months & (1) (2) (3) (5) (7) \\
\hline Thani et al. [34] & 2019 & Qatar & Prediabetes & $57 / 75$ & $\begin{array}{l}\text { I: } 45.51 \pm 8.96 \mathrm{C}: \\
\quad 44.89 \pm 8.88\end{array}$ & $\mathrm{VD}_{3} 30,000 \mathrm{IU} /$ week & placebo & 6 months & (1) (2) (3) (4) (5) (6) (7) \\
\hline $\begin{array}{l}\text { Wallace et al. } \\
\quad[35]\end{array}$ & 2019 & UK & Prediabetes & $35 / 31$ & I: $\begin{aligned} 52.4 & \pm 2.0 \mathrm{C}: 54.0 \\
& \pm 1.7\end{aligned}$ & $\mathrm{VD}_{3} 3000 \mathrm{IU} /$ day & placebo & 26 weeks & (1) (2) (3) (4) (5) (6) (7) \\
\hline
\end{tabular}


Table 1. Cont

\begin{tabular}{|c|c|c|c|c|c|c|c|c|c|}
\hline Study & Year & Country & Participants & $N(\mathrm{I} / \mathrm{C})$ & Age (Years) & Intervention & Control & Duration Period & Outcomes \\
\hline Lu et al. [36] & 2019 & China & Prediabetes & $64 / 65$ & I: $\begin{aligned} 59.29 & \pm 6.07 \mathrm{C}: \\
& 59.41 \pm 9.10\end{aligned}$ & VD $400 \mathrm{IU} /$ day & nothing & 1 year & (1) (2) (3) (5) (6) \\
\hline $\begin{array}{c}\text { Ahmed et al. } \\
\text { [37] }\end{array}$ & 2020 & India & Prediabetes & $52 / 49$ & I: $41.1 \pm 8$ C: $41.6 \pm 7$ & $\mathrm{VD}_{3} 60,000 \mathrm{IU} /$ week & placebo & 12 weeks & (1) (2) (3) (4) (5) (7) \\
\hline Ansari et al. [38] & 2020 & Italy & Prediabetes & $146 / 57$ & NA & $\begin{array}{c}\mathrm{VD}_{3} 50,000 \mathrm{IU} / \text { week for } 2 \text { months, then } \\
\text { twice a month for the next } 2 \text { months, } \\
\text { followed by } 1000 \mathrm{IU} / \text { day for the last } 2 \\
\text { months }\end{array}$ & nothing & 6 months & (2) \\
\hline Bhatt et al. [39] & 2020 & India & Prediabetes & $61 / 60$ & NA & $\mathrm{VD}_{3} 60,000 \mathrm{IU} /$ week $+\mathrm{CaCO}_{3} 1 \mathrm{gm} /$ day & $\begin{array}{c}\text { placebo }+\mathrm{CaCO}_{3} 1 \\
\mathrm{gm} / \mathrm{day} \\
\text { omega-3 } 1000 \mathrm{mg}\end{array}$ & 78 weeks & (1) (2) (3) (5) (7) \\
\hline $\begin{array}{l}\text { Rajabi-Naeeni } \\
\text { et al. [40] }\end{array}$ & 2020 & Iran & Prediabetes & $84 / 84$ & $\begin{array}{l}\text { I: } 39.46 \pm 6.91 \mathrm{C}: \\
\quad 40.82 \pm 7.19\end{array}$ & $\begin{array}{l}\mathrm{VD}_{3} 60,000 \mathrm{IU} / 2 \text { weeks + omega-3 } 1000 \mathrm{mg} \\
\text { twice/day or not }\end{array}$ & $\begin{array}{l}\text { twice/day or } \\
\text { omega-3 placebo }+ \\
\mathrm{VD}_{3} \text { placebo }\end{array}$ & 8 weeks & (1) (2) (5) (6) (7) \\
\hline
\end{tabular}

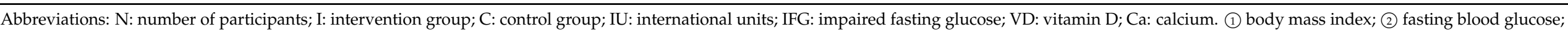

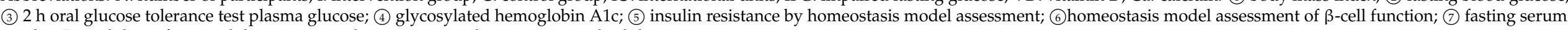
insulin. Partial data of age and duration period were expressed as mean \pm standard deviation.

Table 2. Subgroup analyses.

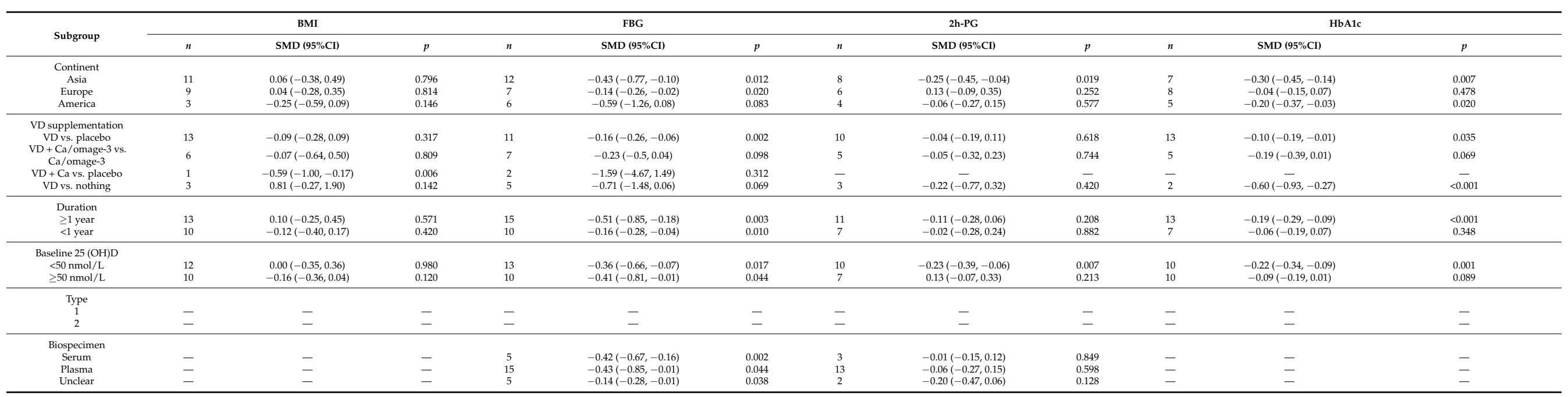


Table 2. Cont.

\begin{tabular}{|c|c|c|c|c|c|c|c|c|c|}
\hline \multirow{2}{*}{ Subgroup } & \multicolumn{3}{|c|}{ HOMA-IR } & \multicolumn{3}{|c|}{ HOMA-B } & \multicolumn{3}{|c|}{ FINS } \\
\hline & $n$ & SMD $(95 \% \mathrm{CI})$ & $p$ & $n$ & SMD ( $95 \% \mathrm{CI})$ & $p$ & $n$ & SMD $(95 \% \mathrm{Cl})$ & $p$ \\
\hline Continent & & & & & & & & & \\
\hline Asia & 11 & $0.08(-0.55,0.72)$ & 0.799 & 6 & $0.22(-0.23,0.67)$ & 0.347 & 7 & $-0.28(-0.43,-0.14)$ & $<0.001$ \\
\hline $\begin{array}{l}\text { Europe } \\
\text { America }\end{array}$ & 6 & $-0.17(-0.55,0.20)$ & 0.361 & 2 & $0.20(-0.16,0.55)$ & 0.278 & 5 & $-0.22(-0.35,-0.09)$ & 0.001 \\
\hline America & & $-0.75(-1.84,0.33)$ & 0.172 & & $0.20(-0.09,0.50)$ & & 3 & $0.05(-0.13,0.22)$ & 0.584 \\
\hline $\begin{array}{l}\text { VD supplementation } \\
\text { VD vs. placebo }\end{array}$ & 9 & $-0.23(-0.46,0.00)$ & 0.054 & 5 & $0.05(-0.24,0.34)$ & 0.718 & 9 & $-0.23(-0.34,-0.13)$ & $<0.001$ \\
\hline $\mathrm{VD}+\mathrm{Ca} /$ omage- $3 \mathrm{vs}$ & 7 & $0.60(-0.28,1.49)$ & 0.181 & 3 & $0.39(-0.32,1.10)$ & 0.286 & 4 & $-0.16(-0.35,0.03)$ & 0.093 \\
\hline $\begin{array}{c}\text { Ca/omage-3 } \\
\text { VD+Ca vs lyacebo }\end{array}$ & & $-1.62(-4.94 .169)$ & 0.337 & - & (a) & & & $0.66(-015027)$ & \\
\hline $\begin{array}{l}\text { +Cavs.spaceoo } \\
\text { VD vs. nothing }\end{array}$ & 3 & $\begin{array}{l}-1.02(-4.94,1.69) \\
-0.75(-1.67,0.17)\end{array}$ & $\begin{array}{l}0.337 \\
0.110\end{array}$ & 2 & $0.31(-0.57,1.18)$ & $0 . \overline{492}$ & 1 & $\begin{array}{l}0.06(-0.1 .1,0.27) \\
-0.52(-1.08,0.04)\end{array}$ & $\begin{array}{l}0.564 \\
0.069\end{array}$ \\
\hline $\begin{array}{l}\text { Duration } \\
\text { s lioar }\end{array}$ & & & & & & & & & \\
\hline $\begin{array}{l}\geq 1 \text { year } \\
<1 \text { year }\end{array}$ & $\begin{array}{c}13 \\
8\end{array}$ & $\begin{array}{c}-0.44(-0.81,-0.06) \\
0.35(-0.42,1.12)\end{array}$ & $\begin{array}{l}0.023 \\
0.374\end{array}$ & $\begin{array}{l}7 \\
3\end{array}$ & $\begin{array}{c}0.34(0.01,0.66) \\
-0.23(-0.49,0.03)\end{array}$ & $\begin{array}{l}0.041 \\
0.084\end{array}$ & $\begin{array}{l}8 \\
7\end{array}$ & $\begin{array}{l}-0.16(-0.26,-0.05) \\
-0.22(-0.36,-0.08)\end{array}$ & $\begin{array}{l}0.004 \\
0.003\end{array}$ \\
\hline Baseline $25(\mathrm{OH}) \mathrm{D}$ & & & & & & & & & \\
\hline $\begin{array}{l}<50 \mathrm{nmol} / \mathrm{L} \\
\geq 50 \mathrm{nmol} / \mathrm{L}\end{array}$ & $\begin{array}{c}10 \\
9\end{array}$ & $\begin{array}{c}0.29(-0.31,0.89) \\
-0.50(-0.96,-0.03)\end{array}$ & $\begin{array}{l}0.346 \\
0.035\end{array}$ & $\begin{array}{l}4 \\
5\end{array}$ & $\begin{array}{c}-0.06(-0.34,0.23) \\
0.27(-0.16,0.71)\end{array}$ & $\begin{array}{l}0.688 \\
0.214\end{array}$ & 5 & $\begin{array}{l}-0.19(-0.33,-0.05) \\
-0.25(-0.38,-0.13)\end{array}$ & $\begin{array}{l}0.008 \\
<0.001\end{array}$ \\
\hline & & & & & & & & & \\
\hline 1 & 18 & $-0.16(-0.56,0.24)$ & 0.441 & 6 & $0.35(-0.08,0.78)$ & 0.109 & - & - & - \\
\hline Biospecimen & & & & & & & & & \\
\hline $\begin{array}{l}\text { Bospecimen } \\
\text { Serum }\end{array}$ & - & - & - & - & - & - & 12 & $-0.23(-0.33,-0.13)$ & $<0.001$ \\
\hline $\begin{array}{c}\text { Plasma } \\
\text { Unclear }\end{array}$ & - & $=$ & $\overline{-}$ & 二 & $=$ & z & ${ }_{1}^{2}$ & $\begin{array}{l}-0.19(-0.47,0.10) \\
0.06(-0.15,0.27)\end{array}$ & $\begin{array}{l}0.205 \\
0.564\end{array}$ \\
\hline
\end{tabular}

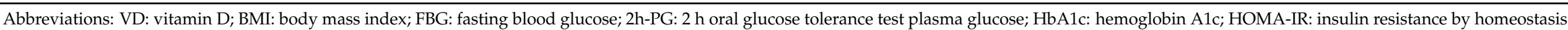
model assessment; HOMA-B: homeostasis model assessment of $\beta$-cell function; FINS: fasting insulin. 


\subsubsection{FBG}

Twenty-five trials involving 3144 participants were included to demonstrate the effect of vitamin D supplementation on FBG in prediabetics [12-21,23,26,27,29-40]. Overall, a significant decrease in FBG content was observed in the vitamin D supplementation group (SMD $=-0.38 ; 95 \% \mathrm{CI}:-0.59,-0.16 ; p=0.001$ ) (Figure 2), with a high heterogeneity $\left(\mathrm{I}^{2}=87.6 \%, p<0.001\right)$. Subgroup analyses suggested similar findings in the following subgroups: Asia, Europe, vitamin D vs. placebo, study duration $\geq 1$ year, study duration $<1$ year, baseline $25(\mathrm{OH}) \mathrm{D}<50 \mathrm{nmol} / \mathrm{L}$, and baseline $25(\mathrm{OH}) \mathrm{D} \geq 50 \mathrm{nmol} / \mathrm{L}$ (Table 2).

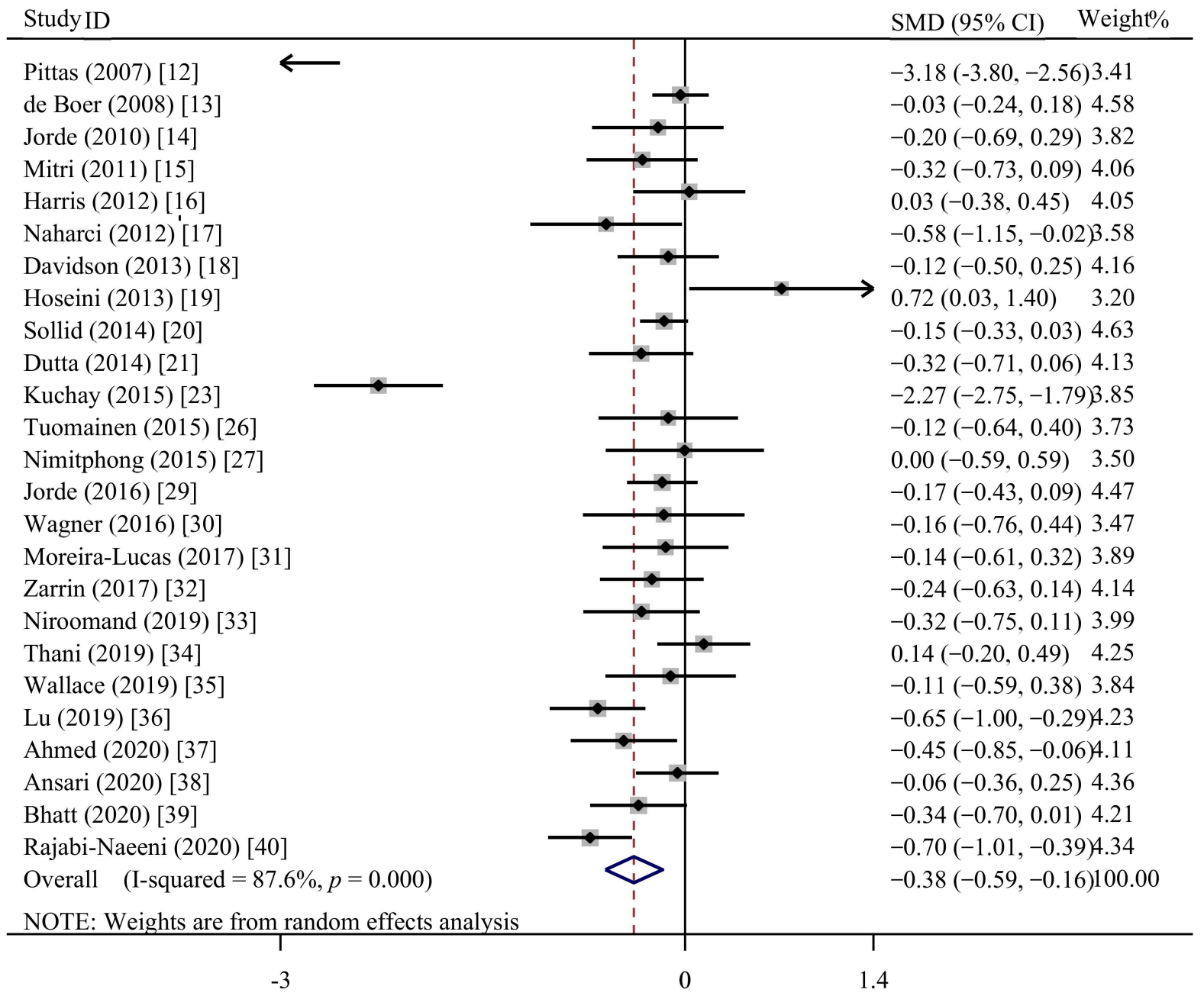

Figure 2. Effect of vitamin D supplementation on fasting blood glucose in prediabetes.

\subsubsection{2h-PG}

Changes in 2h-PG were assessed in 18 studies ( $N=2140)$ [14-16,18,20,21,23,26,27,29-31,33-37,39]. Overall, there was no significant difference in $2 \mathrm{~h}-\mathrm{PG}$ reduction between vitamin $\mathrm{D}$ supplementation and control groups (SMD $=-0.08 ; 95 \% \mathrm{CI}:-0.22,0.06 ; p=0.263$ ) (Figure S3). The heterogeneity was moderate $\left(\mathrm{I}^{2}=56.1 \%, p=0.002\right)$. Nevertheless, effects in the Asia and baseline $25(\mathrm{OH}) \mathrm{D}<50 \mathrm{nmol} / \mathrm{L}$ subgroups significantly showed a remarkably decrease in the intervention group (Table 2). 


\subsection{4. $\mathrm{HbA1c}$}

The effect of vitamin D supplementation on $\mathrm{HbA} 1 \mathrm{c}$ was illustrated in 20 studies $(N=2470)[14-16,18-21,23-32,34,35,37]$. A larger reduction in HbA1c content was found in prediabetes with vitamin D supplementation than in controls $(\mathrm{SMD}=-0.14 ; 95 \% \mathrm{CI}:-0.22$, $-0.06 ; p=0.001)$ (Figure 3), although there was a moderate heterogeneity $\left(\mathrm{I}^{2}=46.5 \%\right.$, $p=0.012$ ). The effects shown in Asia, America, vitamin $\mathrm{D}$ vs. placebo, vitamin $\mathrm{D}$ vs. nothing, study duration $\geq 1$ year, and baseline $25(\mathrm{OH}) \mathrm{D}<50 \mathrm{nmol} / \mathrm{L}$ participants were significantly in favor of the vitamin D supplementation (Table 2).

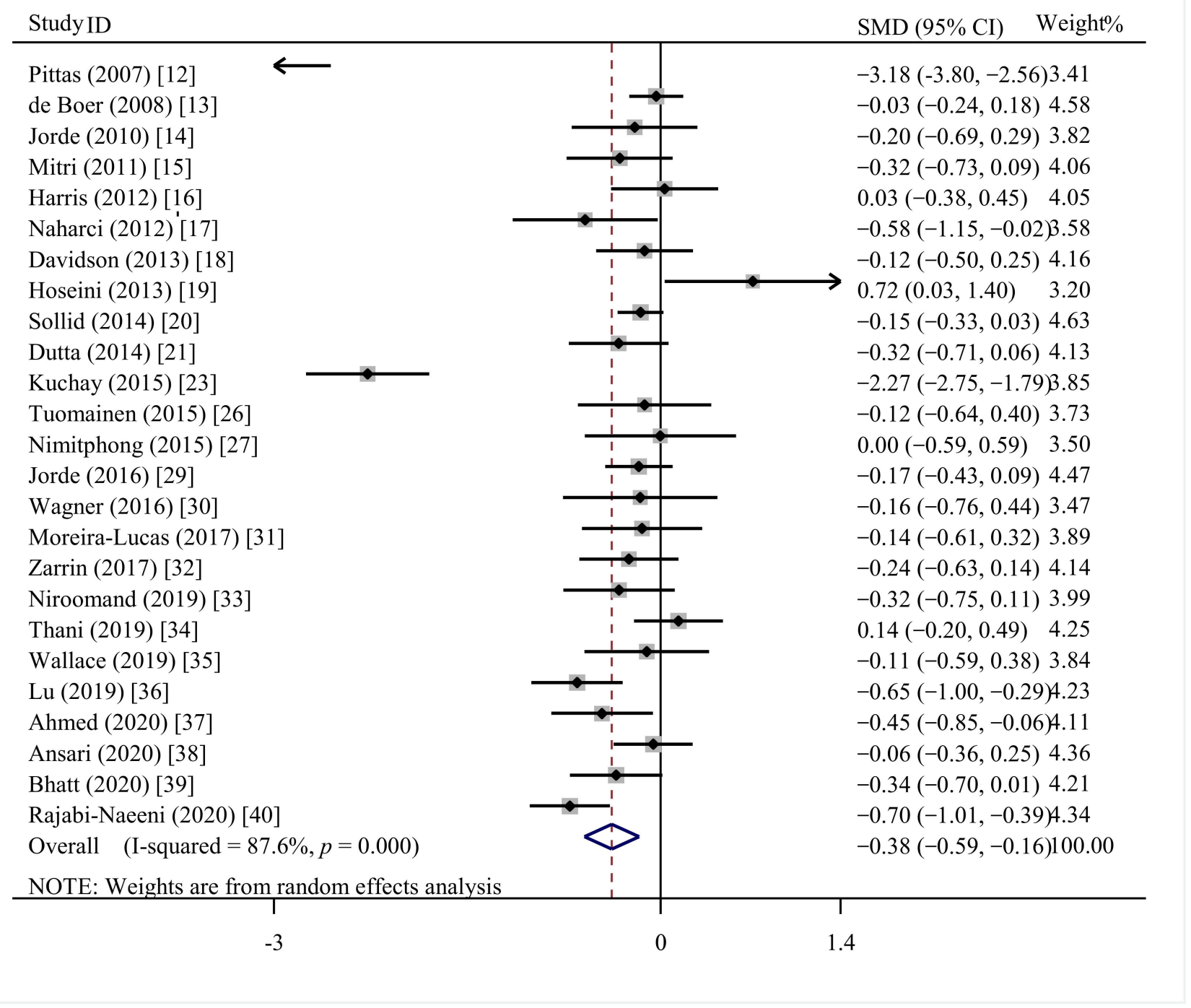

Figure 3. Effect of vitamin D supplementation on glycosylated hemoglobin A1c in prediabetes.

\subsubsection{HOMA-IR}

There were $21(N=2748)$ out of 29 RCTs that reported HOMA-IR [12-14,16-22,26,27,29,32-37,39,40]. Results showed that vitamin D supplementation had no significant impact on HOMA-IR (SMD $=-0.15$; 95\%CI: $-0.50,0.20 ; p=0.402$ ) (Figure S4), with the heterogeneity relatively high $\left(\mathrm{I}^{2}=94.8 \%, p<0.001\right.$ ). However, the following subgroups favor the vitamin $D$ supplementation group: study duration $\geq 1$ year and baseline $25(\mathrm{OH}) \mathrm{D} \geq 50 \mathrm{nmol} / \mathrm{L}$ (Table 2). 


\subsubsection{HOMA-B}

The results of 10 clinical trials $(N=928)$ revealed that treatment with vitamin $\mathrm{D}$ failed to restore HOMA-B (SMD $=0.19$; $95 \% \mathrm{CI}:-0.09,0.47 ; p=0.179)$; the results showed high heterogeneity $\left(\mathrm{I}^{2}=75.9 \%, p<0.001\right)$ (Figure S5) $[18,19,21,26,27,31,34-36,40]$. The subgroup of study duration $\geq 1$ year was significantly in favor of vitamin $D$ supplementation (Table 2 ).

\subsubsection{FINS}

Fourteen trials encompassing 2172 participants were included [13,16,17,20,26,29,31-35,37,39,40]. The present meta-analysis found that vitamin $\mathrm{D}$ supplementation had a beneficial effect on FINS in prediabetics (SMD $=0.18 ; 95 \% \mathrm{CI}:-0.26,-0.09 ; p<0.001$, with low heterogeneity $\left(\mathrm{I}^{2}=20.7 \%\right.$, $p=0.223$ ) (Figure 4). The subgroup of Asia, Europe, vitamin D vs. placebo, study duration $\geq 1$ year, study duration $<1$ year, and baseline $25(\mathrm{OH}) \mathrm{D}<50 \mathrm{nmol} / \mathrm{L}$ showed similar results (Table 2).

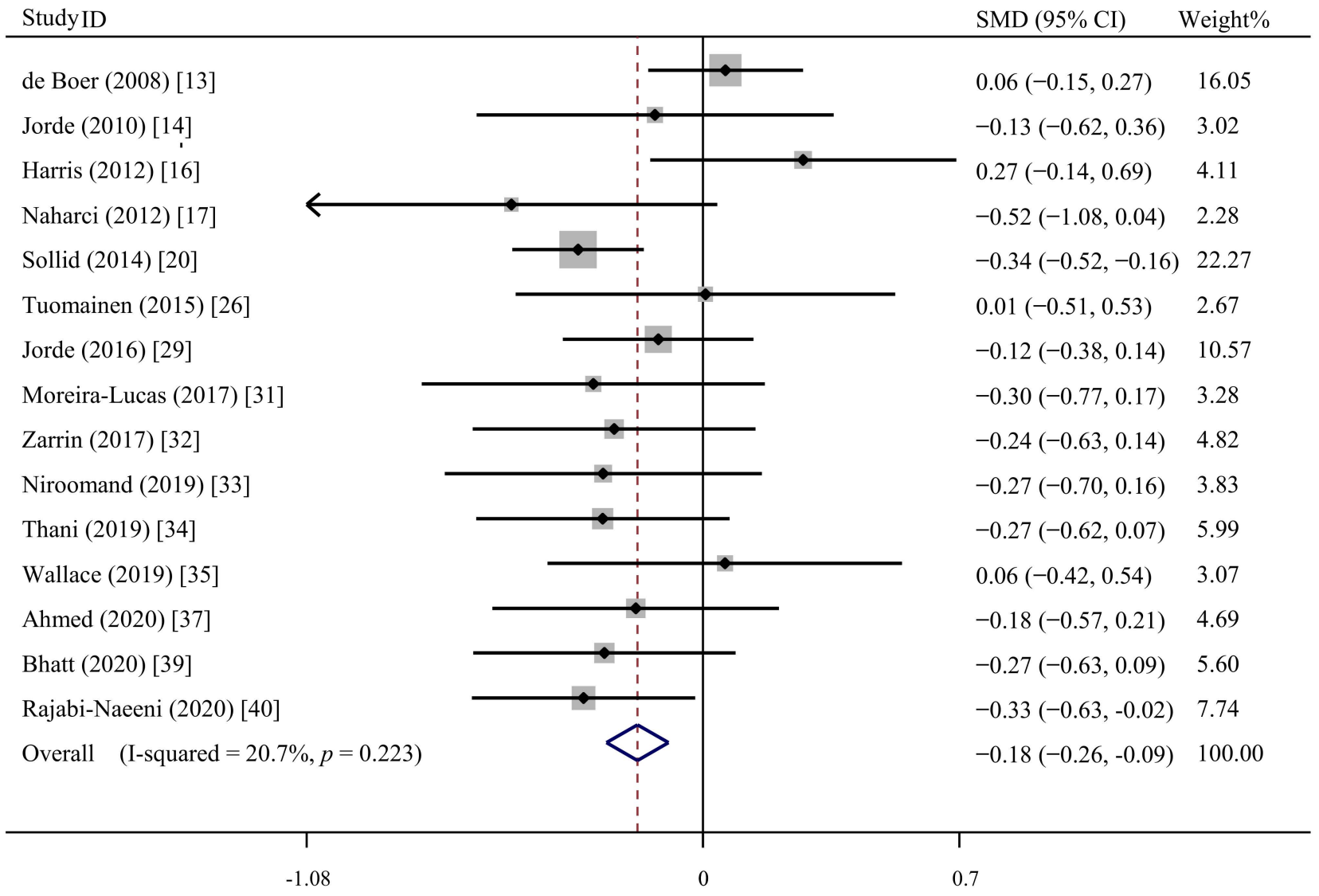

Figure 4. Effect of vitamin D supplementation on fasting serum insulin in prediabetes.

\subsection{Meta-Regression}

To uncover the potential causes of high heterogeneity, a meta-regression analysis was performed involving publication year, geographic locations, vitamin D supplementation, study duration, and baseline $25(\mathrm{OH}) \mathrm{D}$. None were found to contribute to the heterogeneity between studies. 


\subsection{Sensitivity Analysis}

In the sensitivity analysis, the results of BMI, 2h-PG, and HOMA-B levels remained basically robust. The remarkable effect of vitamin $\mathrm{D}$ supplementation on $\mathrm{HbA1c}$ disappeared when excluding Kuchey et al. [23], and a similar trend was found for FINS when excluding de Boer et al. [13] and Sollid et al. [20], as the heterogeneity decreased to $0.00 \%$. However, the effect of vitamin D on HOMA-IR in prediabetes turned to significant (SMD $=-0.23$; $95 \% \mathrm{CI}:-0.42,-0.03 ; p<0.001)$, with high heterogeneity $\left(\mathrm{I}^{2}=82.5 \%, p<0.001\right)$, when excluding Pittas et al. [12] and Bhatt et al. [39]. In addition, by excluding Pittas et al. [12] and Kuchey et al. [23] from the meta-analysis, the heterogeneity for the beneficial effect of vitamin D supplementation on FBG decreased from $87.6 \%$ to $41.5 \%$.

\subsection{Publication Bias}

The funnel plot showed that the study distribution was basically symmetrical (Figure 5), and statistical tests showed no evidence of a publication bias (Begg's test: $p=0.151,95 \% \mathrm{CI}$ : $-5.76,0.94)$.

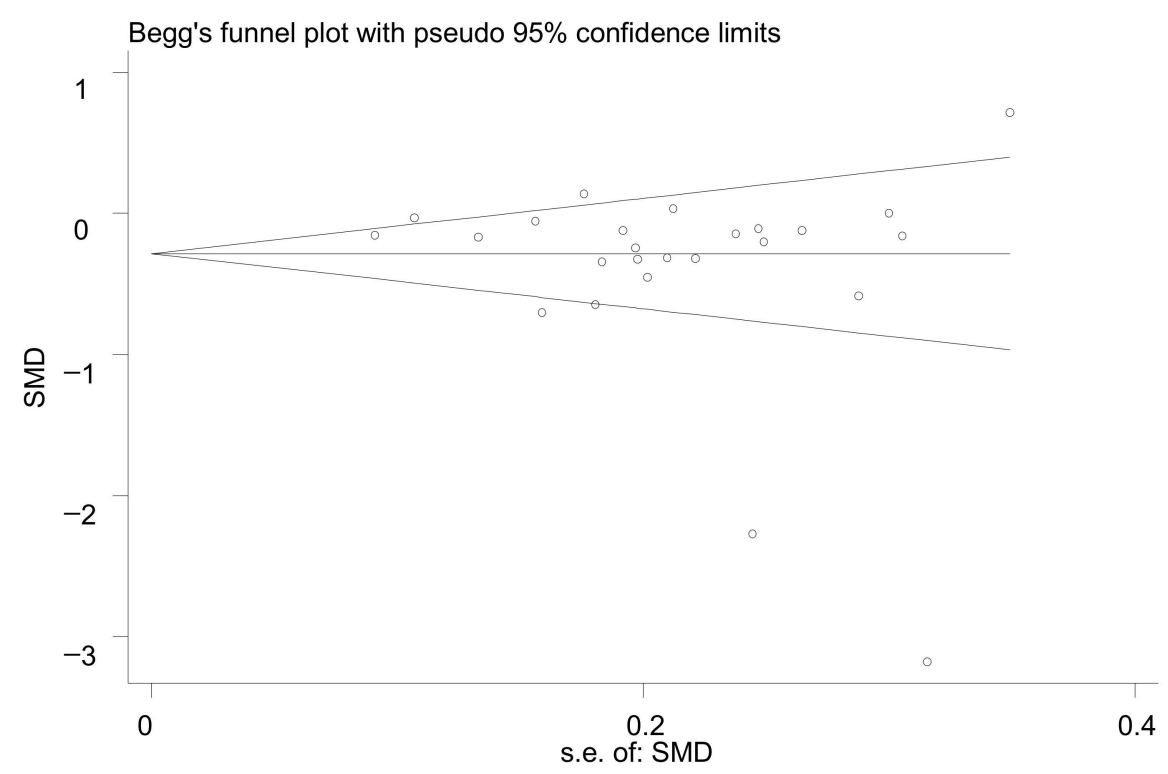

Figure 5. Funnel plot of fasting blood glucose changes.

\section{Discussion}

This meta-analysis showed a positive impact of vitamin D supplementation on FBG, $\mathrm{HbA1c}$, and FINS in prediabetes but showed no benefit of vitamin D supplementation with regard to improving BMI, 2h-PG, HOMA-IR, and HOMA-B.

The results of the present research are consistent with Poolsup et al. [7] in terms of FBG, 2h-PG, HbA1c, and HOMA-IR, while the results for HOMA-IR are partially consistent with $\mathrm{Yu}$ et al. [6]. The present analysis contained the most comprehensive review of trials available compared with previous meta-analyses [6,7]. In addition to the indicators reported in previous studies (e.g., FBG, 2h-PG, HbA1c, HOMA-IR), we added the following indexes to better reflect the control of FBG and the protection of islets in prediabetes by vitamin D supplementation: HOMA-B, FINS, and BMI.

We found that vitamin $\mathrm{D}$ supplementation generated various effects among different continents: significant reductions in FBG, 2h-PG, HbA1c, and FINS levels were seen in Asian populations, FBG and FINS reductions were found in Europeans, and decreased $\mathrm{HbA1c}$ was detected in Americans, with the same index falling even more sharply in Asians. Asians showed a higher level of free 25(OH)D than the Europeans and Americans after the same dose of vitamin $\mathrm{D}_{3}$ supplementation, which may reflect the discrepancy in the affinity of vitamin $\mathrm{D}$ binding protein to $25(\mathrm{OH}) \mathrm{D}$ [41]. In addition to vitamin D binding 
protein, cytochrome P450 family 2 subfamily R polypeptide 1 (CYP2R1) and vitamin D receptor $(V D R)$ also affected the vitamin D status, metabolism, and function. Jones et al. [42] reported that the frequencies of $C Y P 2 R 1$ and $V D R$ variants differed by geographic areas. Elkum et al. [43] found CYP2R1 genes associated with vitamin D status in Arabs (CYP2R1 rs10741657, rs10500804 and rs12794714) and South Asians (CYP2R1 rs10741657) but not Southeast Asians. Lu et al. [44] confirmed that CYP2R1 rs2060793 variant was associated with serum 25(OH)D levels in the Shanghai but not the Beijing subpopulation. The VDR were similar to $C Y P 2 R 1$ genes, showing differences between races [45-47]. In addition, Rajan et al. and Weishaar et al. confirmed that people with darker skin color (Asians' skin was noted as darker than Europeans and Americans) were independently and significantly associated with poor vitamin D status, which may be related to better FBG improvement in Asians [48,49].

The results of the duration of the intervention were inconsistent with previous studies [7]. Perhaps due to the small sample size, the meta-analysis of Poolsup et al. did not reached a conclusion in relation to study duration and vitamin $\mathrm{D}$ supplementation effect. This analysis included 19 additional studies and revealed that interventions of vitamin $\mathrm{D} \geq 1$ year were able to improve the contents of FBG, HbA1c, HOMA-IR, HOMA-B, and FINS in prediabetes; FBG and FINS in patients with prediabetes were also improved when intervention durations were $<1$ year. Previous studies [50,51] found that the protective effects of vitamin D supplementation on T2DM were primarily generated by intervention periods of less than three months. The reason for this was not only the difference in dosage; the progression of diabetes could also weaken the effect of long-term intervention over time. Prediabetes is a reversible process, but T2DM is not [52]. Therefore, the longer the vitamin D supplementation in prediabetes, the more effective the prevention of the onset of diabetes and the less likely the disease will progress.

A cross-sectional analysis conducted by the U.S. National Health and Nutrition Examination Survey (2007-2012) found that serum 25(OH)D levels from deficient $(<50 \mathrm{nmol} / \mathrm{L})$ or insufficient (50.1-74.9 nmol/L) individuals were more likely to suffer from prediabetes compared to sufficient individuals ( $>75 \mathrm{nmol} / \mathrm{L}$ ) [53]. In addition, studies have also shown that $25(\mathrm{OH}) \mathrm{D}$ levels are inversely associated with the prevalence of diabetes and HOMAIR [54,55]. Subgroup analysis related to baseline 25(OH)D levels showed that, in subjects with baseline $25(\mathrm{OH}) \mathrm{D}<50 \mathrm{nmol} / \mathrm{L}$, treatment of vitamin D significantly improved FBG, 2h-PG, HbA1c, and FINS; FBG showed a better improvement effect among subjects with baseline $25(\mathrm{OH}) \mathrm{D} \geq 50 \mathrm{nmol} / \mathrm{L}$. In addition, there was also a notably ameliorate effect on HOMA-IR. Indicators of improvement were not entirely consistent in participants with different baseline 25(OH)D levels; a possible reason for this is that vitamin D could directly act on the receptors on the pancreas to improve islet function, while the mechanism of insulin resistance is more complicated and requires longer intervention to achieve the effect [56].

First, to our knowledge, this meta-analysis is unique, as more indicators were used to elaborate the effects of vitamin D supplementation on prediabetes. Second, the Cochrane Collaboration tool was used to evaluate the quality of the trials. Third, we explored influential factors, including continents, intervention strategies, intervention durations, baseline $25(\mathrm{OH}) \mathrm{D}$, and different calculating methods (specifically applicable to HOMA-IR and HOMA-B). Lastly, only RCTs with oral vitamin D supplementation were included in the meta-analysis. However, the heterogeneity of the results was still significant because of discrepancies in vitamin D types, doses, study durations, participants, and some unknown factors. Other limitations were that most of containing studies did not evaluate the effects of sun exposure, dietary intake, and physical exercise, as different lifestyles and genes between studies may affect vitamin status [57-59]. Third, the intervention and control groups were unbalanced; some control groups from different studies were without a matching placebo $[17,19,21,23,27,36,38]$, and participants clearly knowing their groupings might result in a Hawthorne effect. Fourth, the biospecimens were nearly all serum and plasma samples (some studies were unclear); subgroup analyses revealed that glucose indexes were not affected by the difference in biospecimens, but there were three studies on 
FINS that were collected either from plasma or otherwise. The specific reason needs to be studied due to the small number of studies. Finally, some studies included two intervention groups; some intervention participants underwent dose changes [17,19,21,23,26-28,33,38], and we were unable to accurately determine the relationship between individual doses and improvement in indicators.

\section{Conclusions}

Oral supplementation of vitamin $\mathrm{D}$ has been shown to exert better effects in improving FBG, $\mathrm{HbA1c}$, and FINS compared with controls among prediabetes; it has not been found to regulate BMI. Long-term vitamin D supplementation could show an additional effect in participants with vitamin D deficiency for 2h-PG, HOMA-IR, and HOMA-B. More large-scaled clinical trials are needed to elucidate the association between vitamin $\mathrm{D}$ and prediabetes.

Supplementary Materials: The following are available online at https:/ / www.mdpi.com/article/10 $.3390 /$ nu13124464/s1, Figure S1: Risk of bias assessment of the included studies using the Cochrane Collaboration tool (A) Risk of bias in included studies; (B) Risk of bias across each bias domain, Figure S2: Effect of vitamin D supplementation on body mass index in prediabetes, Figure S3: Effect of vitamin D supplementation on $2 \mathrm{~h}$ oral glucose tolerance test plasma glucose in prediabetes, Figure S4: Effect of vitamin D supplementation on insulin resistance by homeostasis model assessment in prediabetes, Figure S5: Effect of vitamin D supplementation on homeostasis model assessment of $\beta$-cell function in prediabetes.

Author Contributions: Research design, Y.Z. and W.L.; Data collection, Y.Z. and Y.X.; Data analysis, Y.X., D.Z. and Y.L.; Funding acquisition, W.L. and X.L.; Writing-original draft, Y.Z.; Writingreview and editing, Y.Z., Z.X., J.G. and X.L. All authors have read and agree to the published version of the manuscript.

Funding: This research was funded by the National Natural Science Foundation of China (No. 81872626, 82003454), Chinese Nutrition Society-Bright Moon Seaweed Group Nutrition and Health Research Fund (No. CNS-BMSG2020A63); Chinese Nutrition Society-Zhendong National Physical Fitness and Health Research Fund (No. CNS-ZD2019066), and Key R\&D and Promotion Projects in Henan Province (No. 212102310219).

Data Availability Statement: All data relevant to the study are included in the article.

Conflicts of Interest: The authors confirm that they have no conflict of interest to report.

\section{References}

1. Saeedi, P.; Petersohn, I.; Salpea, P.; Malanda, B.; Karuranga, S.; Unwin, N.; Colagiuri, S.; Guariguata, L.; Motala, A.A.; Ogurtsova, K.; et al. Global and regional diabetes prevalence estimates for 2019 and projections for 2030 and 2045: Results from the International Diabetes Federation Diabetes Atlas, 9(th) edition. Diabetes Res. Clin. Pract. 2019, 157, 107843. [CrossRef] [PubMed]

2. Tabak, A.G.; Herder, C.; Rathmann, W.; Brunner, E.J.; Kivimaki, M. Prediabetes: A high-risk state for diabetes development. Lancet 2012, 379, 2279-2290. [CrossRef]

3. Lim, S.; Kim, M.J.; Choi, S.H.; Shin, C.S.; Park, K.S.; Jang, H.C.; Billings, L.K.; Meigs, J.B. Association of vitamin D deficiency with incidence of type 2 diabetes in high-risk Asian subjects. Am. J. Clin. Nutr. 2013, 97, 524-530. [CrossRef] [PubMed]

4. Dhas, Y.; Banerjee, J.; Damle, G.; Mishra, N. Association of vitamin D deficiency with insulin resistance in middle-aged type 2 diabetics. Clin. Chim. Acta 2019, 492, 95-101. [CrossRef] [PubMed]

5. Gao, Y.; Zheng, T.; Ran, X.; Ren, Y.; Chen, T.; Zhong, L.; Yan, D.; Yan, F.; Wu, Q.; Tian, H. Vitamin D and Incidence of Prediabetes or Type 2 Diabetes: A Four-Year Follow-Up Community-Based Study. Dis. Markers 2018, 2018, 1926308. [CrossRef] [PubMed]

6. Yu, L.; Zhai, Y.; Shen, S. Association between vitamin D and prediabetes: A PRISMA-compliant meta-analysis. Medicine 2020, 99, e19034. [CrossRef]

7. Poolsup, N.; Suksomboon, N.; Plordplong, N. Effect of vitamin D supplementation on insulin resistance and glycaemic control in prediabetes: A systematic review and meta-analysis. Diabet. Med. 2016, 33, 290-299. [CrossRef]

8. American Diabetes, A. 2. Classification and Diagnosis of Diabetes: Standards of Medical Care in Diabetes-2020. Diabetes Care 2020, 43, S14-S31. [CrossRef] [PubMed]

9. Consultation, W.I. Definition and Diagnosis of Diabetes and Intermediate Hyperglycaemia; World Health Organization: Geneva, Switzerland, 2006. 
10. Higgins, J.P.; Altman, D.G.; Gotzsche, P.C.; Juni, P.; Moher, D.; Oxman, A.D.; Savovic, J.; Schulz, K.F.; Weeks, L.; Sterne, J.A.; et al. The Cochrane Collaboration's tool for assessing risk of bias in randomised trials. BMJ 2011, 343, d5928. [CrossRef] [PubMed]

11. Borenstein, M.; Hedges, L.V.; Higgins, J.P.; Rothstein, H.R. A basic introduction to fixed-effect and random-effects models for meta-analysis. Res. Synth. Methods 2010, 1, 97-111. [CrossRef] [PubMed]

12. Pittas, A.G.; Harris, S.S.; Stark, P.C.; Dawson-Hughes, B. The effects of calcium and vitamin D supplementation on blood glucose and markers of inflammation in nondiabetic adults. Diabetes Care 2007, 30, 980-986. [CrossRef] [PubMed]

13. De Boer, I.H.; Tinker, L.F.; Connelly, S.; Curb, J.D.; Howard, B.V.; Kestenbaum, B.; Larson, J.C.; Manson, J.E.; Margolis, K.L.; Siscovick, D.S.; et al. Calcium plus vitamin D supplementation and the risk of incident diabetes in the Women's Health Initiative. Diabetes Care 2008, 31, 701-707. [CrossRef] [PubMed]

14. Jorde, R.; Sneve, M.; Torjesen, P.; Figenschau, Y. No improvement in cardiovascular risk factors in overweight and obese subjects after supplementation with vitamin D3 for 1 year. J. Intern. Med. 2010, 267, 462-472. [CrossRef] [PubMed]

15. Mitri, J.; Dawson-Hughes, B.; Hu, F.B.; Pittas, A.G. Effects of vitamin D and calcium supplementation on pancreatic beta cell function, insulin sensitivity, and glycemia in adults at high risk of diabetes: The Calcium and Vitamin D for Diabetes Mellitus (CaDDM) randomized controlled trial. Am. J. Clin. Nutr. 2011, 94, 486-494. [CrossRef] [PubMed]

16. Harris, S.S.; Pittas, A.G.; Palermo, N.J. A randomized, placebo-controlled trial of vitamin D supplementation to improve glycaemia in overweight and obese African Americans. Diabetes Obes. Metab. 2012, 14, 789-794. [CrossRef] [PubMed]

17. Naharci, I.; Bozoglu, E.; Kocak, N.; Doganci, S.; Doruk, H.; Serdar, M. Effect of vitamin D on insulin sensitivity in elderly patients with impaired fasting glucose. Geriatr. Gerontol. Int. 2012, 12, 454-460. [CrossRef] [PubMed]

18. Davidson, M.B.; Duran, P.; Lee, M.L.; Friedman, T.C. High-dose vitamin D supplementation in people with prediabetes and hypovitaminosis D. Diabetes Care 2013, 36, 260-266. [CrossRef]

19. Hoseini, S.A.; Aminorroaya, A.; Iraj, B.; Amini, M. The effects of oral vitamin D on insulin resistance in pre-diabetic patients. J. Res. Med. Sci. 2013, 18, 47-51.

20. Sollid, S.T.; Hutchinson, M.Y.; Fuskevag, O.M.; Figenschau, Y.; Joakimsen, R.M.; Schirmer, H.; Njolstad, I.; Svartberg, J.; Kamycheva, E.; Jorde, R. No effect of high-dose vitamin D supplementation on glycemic status or cardiovascular risk factors in subjects with prediabetes. Diabetes Care 2014, 37, 2123-2131. [CrossRef]

21. Dutta, D.; Mondal, S.A.; Choudhuri, S.; Maisnam, I.; Hasanoor Reza, A.H.; Bhattacharya, B.; Chowdhury, S.; Mukhopadhyay, S. Vitamin-D supplementation in prediabetes reduced progression to type 2 diabetes and was associated with decreased insulin resistance and systemic inflammation: An open label randomized prospective study from Eastern India. Diabetes Res. Clin. Pract. 2014, 103, e18-e23. [CrossRef] [PubMed]

22. Oosterwerff, M.M.; Eekhoff, E.M.; Van Schoor, N.M.; Boeke, A.J.; Nanayakkara, P.; Meijnen, R.; Knol, D.L.; Kramer, M.H.; Lips, P. Effect of moderate-dose vitamin D supplementation on insulin sensitivity in vitamin D-deficient non-Western immigrants in the Netherlands: A randomized placebo-controlled trial. Am. J. Clin. Nutr. 2014, 100, 152-160. [CrossRef] [PubMed]

23. Kuchay, M.S.; Laway, B.A.; Bashir, M.I.; Wani, A.I.; Misgar, R.A.; Shah, Z.A. Effect of Vitamin D supplementation on glycemic parameters and progression of prediabetes to diabetes: A 1-year, open-label randomized study. Indian J. Endocrinol. Metab. 2015, 19, 387-392. [PubMed]

24. Barengolts, E.; Manickam, B.; Eisenberg, Y.; Akbar, A.; Kukreja, S.; Ciubotaru, I. Effect of High-Dose Vitamin D Repletion on Glycemic Control in African-American Males with Prediabetes and Hypovitaminosis D. Endocr. Pract. 2015, $21,604-612$. [CrossRef] [PubMed]

25. Didriksen, A.; Burild, A.; Jakobsen, J.; Fuskevag, O.M.; Jorde, R. Vitamin D3 increases in abdominal subcutaneous fat tissue after supplementation with vitamin D3. Eur. J. Endocrinol. 2015, 172, 235-241. [CrossRef] [PubMed]

26. Tuomainen, T.P.; Virtanen, J.K.; Voutilainen, S.; Nurmi, T.; Mursu, J.; de Mello, V.D.; Schwab, U.; Hakumaki, M.; Pulkki, K.; Uusitupa, M. Glucose Metabolism Effects of Vitamin D in Prediabetes: The VitDmet Randomized Placebo-Controlled Supplementation Study. J. Diabetes Res. 2015, 2015, 672653. [CrossRef] [PubMed]

27. Nimitphong, H.; Samittarucksa, R.; Saetung, S.; Bhirommuang, N.; Chailurkit, L.O.; Ongphiphadhanakul, B. The Effect of Vitamin D Supplementation on Metabolic Phenotypes in Thais with Prediabetes. J. Med. Assoc. Thai. 2015, 98, 1169-1178.

28. Forouhi, N.G.; Menon, R.K.; Sharp, S.J.; Mannan, N.; Timms, P.M.; Martineau, A.R.; Rickard, A.P.; Boucher, B.J.; Chowdhury, T.A.; Griffiths, C.J.; et al. Effects of vitamin D2 or D3 supplementation on glycaemic control and cardiometabolic risk among people at risk of type 2 diabetes: Results of a randomized double-blind placebo-controlled trial. Diabetes Obes. Metab. 2016, 18, 392-400. [CrossRef]

29. Jorde, R.; Sollid, S.T.; Svartberg, J.; Schirmer, H.; Joakimsen, R.M.; Njolstad, I.; Fuskevag, O.M.; Figenschau, Y.; Hutchinson, M.Y. Vitamin D 20,000 IU per Week for Five Years Does Not Prevent Progression From Prediabetes to Diabetes. J. Clin. Endocrinol. Metab. 2016, 101, 1647-1655. [CrossRef] [PubMed]

30. Wagner, H.; Alvarsson, M.; Mannheimer, B.; Degerblad, M.; Ostenson, C.G. No Effect of High-Dose Vitamin D Treatment on beta-Cell Function, Insulin Sensitivity, or Glucose Homeostasis in Subjects With Abnormal Glucose Tolerance: A Randomized Clinical Trial. Diabetes Care 2016, 39, 345-352. [CrossRef]

31. Moreira-Lucas, T.S.; Duncan, A.M.; Rabasa-Lhoret, R.; Vieth, R.; Gibbs, A.L.; Badawi, A.; Wolever, T.M. Effect of vitamin D supplementation on oral glucose tolerance in individuals with low vitamin D status and increased risk for developing type 2 diabetes (EVIDENCE): A double-blind, randomized, placebo-controlled clinical trial. Diabetes Obes. Metab. 2017, 19 , 133-141. [CrossRef] 
32. Zarrin, R.; Ayremlou, P.; Ghassemi, F. The Effect of Vitamin D Supplementation on the Glycemic Status and the Percentage of Body Fat Mass in Adults with Prediabetes: A Randomized Clinical Trial. Iran. Red Crescent Med. J. 2017, 19, e41718. [CrossRef]

33. Niroomand, M.; Fotouhi, A.; Irannejad, N.; Hosseinpanah, F. Does high-dose vitamin D supplementation impact insulin resistance and risk of development of diabetes in patients with pre-diabetes? A double-blind randomized clinical trial. Diabetes Res. Clin. Pract. 2019, 148, 1-9. [CrossRef] [PubMed]

34. Al Thani, M.; Sadoun, E.; Sofroniou, A.; Jayyousi, A.; Baagar, K.A.M.; Al Hammaq, A.; Vinodson, B.; Akram, H.; Bhatti, Z.S.; Nasser, H.S.; et al. The effect of vitamin D supplementation on the glycemic control of pre-diabetic Qatari patients in a randomized control trial. BMC Nutr. 2019, 5, 46. [CrossRef] [PubMed]

35. Wallace, H.J.; Holmes, L.; Ennis, C.N.; Cardwell, C.R.; Woodside, J.V.; Young, I.S.; Bell, P.M.; Hunter, S.J.; McKinley, M.C. Effect of vitamin D3 supplementation on insulin resistance and beta-cell function in prediabetes: A double-blind, randomized, placebo-controlled trial. Am. J. Clin. Nutr. 2019, 110, 1138-1147. [CrossRef] [PubMed]

36. Lu, J.; Liu, Y.; Zhang, J.; Lou, P.; Liu, Y.; Zhou, Y. Effect of vitamin D intervention on the outcome of glucose metabolism in patients with impaired glucose regulation. Chin. J. Clin. Nutr. 2019, 27, 349-353.

37. Ahmed, M.M.; Zingade, U.S.; Badaam, K.M. Effect of Vitamin D3 Supplementation on Insulin Sensitivity in Prediabetes With Hypovitaminosis D: A Randomized Placebo-Controlled Trial. Cureus 2020, 12, e12009. [CrossRef]

38. Ansari, M.G.A.; Sabico, S.; Clerici, M.; Khattak, M.N.K.; Wani, K.; Al-Musharaf, S.; Amer, O.E.; Alokail, M.S.; Al-Daghri, N.M. Vitamin D Supplementation Is Associated with Increased Glutathione Peroxidase-1 Levels in Arab Adults with Prediabetes. Antioxidants 2020, 9, 118. [CrossRef]

39. Bhatt, S.P.; Misra, A.; Pandey, R.M.; Upadhyay, A.D.; Gulati, S.; Singh, N. Vitamin D Supplementation in Overweight/obese Asian Indian Women with Prediabetes Reduces Glycemic Measures and Truncal Subcutaneous Fat: A 78 Weeks Randomized Placebo-Controlled Trial (PREVENT-WIN Trial). Sci Rep. 2020, 10, 220. [CrossRef] [PubMed]

40. Rajabi-Naeeni, M.; Dolatian, M.; Qorbani, M.; Vaezi, A.A. The effect of omega-3 and vitamin D co-supplementation on glycemic control and lipid profiles in reproductive-aged women with pre-diabetes and hypovitaminosis D: A randomized controlled trial. Diabetol. Metab. Syndr. 2020, 12, 41. [CrossRef] [PubMed]

41. Gopal-Kothandapani, J.S.; Evans, L.F.; Walsh, J.S.; Gossiel, F.; Rigby, A.S.; Eastell, R.; Bishop, N.J. Effect of vitamin D supplementation on free and total vitamin D: A comparison of Asians and Caucasians. Clin. Endocrinol. 2019, 90, 222-231. [CrossRef] [PubMed]

42. Jones, P.; Lucock, M.; Chaplin, G.; Jablonski, N.G.; Veysey, M.; Scarlett, C.; Beckett, E. Distribution of variants in multiple vitamin D-related loci (DHCR7/NADSYN1, GC, CYP2R1, CYP11A1, CYP24A1, VDR, RXRalpha and RXRgamma) vary between European, East-Asian and Sub-Saharan African-ancestry populations. Genes Nutr. 2020, 15, 5. [CrossRef] [PubMed]

43. Elkum, N.; Alkayal, F.; Noronha, F.; Ali, M.M.; Melhem, M.; Al-Arouj, M.; Bennakhi, A.; Behbehani, K.; Alsmadi, O.; Abubaker, J. Vitamin D insufficiency in Arabs and South Asians positively associates with polymorphisms in GC and CYP2R1 genes. PLoS ONE 2014, 9, e113102.

44. Lu, L.; Sheng, H.; Li, H.; Gan, W.; Liu, C.; Zhu, J.; Loos, R.J.; Lin, X. Associations between common variants in GC and DHCR7/NADSYN1 and vitamin D concentration in Chinese Hans. Hum. Genet. 2012, 131, 505-512. [CrossRef] [PubMed]

45. Rivera-Paredez, B.; Macias, N.; Martinez-Aguilar, M.M.; Hidalgo-Bravo, A.; Flores, M.; Quezada-Sanchez, A.D.; Denova-Gutierrez, E.; Cid, M.; Martinez-Hernandez, A.; Orozco, L.; et al. Association between Vitamin D Deficiency and Single Nucleotide Polymorphisms in the Vitamin D Receptor and GC Genes and Analysis of Their Distribution in Mexican Postmenopausal Women. Nutrients 2018, 10, 1175. [CrossRef]

46. Angel, B.; Lera, L.; Marquez, C.; Albala, C. The association of VDR polymorphisms and type 2 diabetes in older people living in community in Santiago de Chile. Nutr. Diabetes 2018, 8, 31. [CrossRef] [PubMed]

47. Rodrigues, K.F.; Pietrani, N.T.; Bosco, A.A.; de Sousa, M.C.R.; Silva, I.F.O.; Silveira, J.N.; Gomes, K.B. Lower Vitamin D Levels, but Not VDR Polymorphisms, Influence Type 2 Diabetes Mellitus in Brazilian Population Independently of Obesity. Medicina 2019, 55, 188. [CrossRef] [PubMed]

48. Rajan, S.; Weishaar, T.; Keller, B. Weight and skin colour as predictors of vitamin D status: Results of an epidemiological investigation using nationally representative data. Public Health Nutr. 2017, 20, 1857-1864. [CrossRef]

49. Weishaar, T.; Rajan, S.; Keller, B. Probability of Vitamin D Deficiency by Body Weight and Race/Ethnicity. J. Am. Board Fam. Med. 2016, 29, 226-232. [CrossRef]

50. Krul-Poel, Y.H.; Ter Wee, M.M.; Lips, P.; Simsek, S. Management of endocrine disease: The effect of vitamin D supplementation on glycaemic control in patients with type 2 diabetes mellitus: A systematic review and meta-analysis. Eur. J. Endocrinol. 2017, 176, R1-R14. [CrossRef] [PubMed]

51. Li, X.; Liu, Y.; Zheng, Y.; Wang, P.; Zhang, Y. The Effect of Vitamin D Supplementation on Glycemic Control in Type 2 Diabetes Patients: A Systematic Review and Meta-Analysis. Nutrients 2018, 10, 375. [CrossRef] [PubMed]

52. Busquets-Cortes, C.; Bennasar-Veny, M.; Lopez-Gonzalez, A.A.; Fresneda, S.; Abbate, M.; Yanez, A.M. Utility of Fatty Liver Index to predict reversion to normoglycemia in people with prediabetes. PLoS ONE 2021, 16, e0249221. [CrossRef] [PubMed]

53. Pojednic, R.M.; Trussler, E.M.; Navon, J.D.; Lemire, S.C.; Siu, E.C.; Metallinos-Katsaras, E.S. Vitamin D deficiency associated with risk of prediabetes among older adults: Data from the National Health and Nutrition Examination Survey (NHANES), $2007-2012$. Diabetes Metab. Res. Rev. 2021, e3499. [CrossRef] [PubMed] 
54. Nakamura, K.; Hui, S.P.; Ukawa, S.; Okada, E.; Nakagawa, T.; Imae, A.; Okabe, H.; Chen, Z.; Miura, Y.; Chiba, H.; et al. Serum 25-hydroxyvitamin D3 Levels and Diabetes in a Japanese Population: The DOSANCO Health Study. J. Epidemiol. 2021. [CrossRef] [PubMed]

55. Schleu, M.F.; Barreto-Duarte, B.; Arriaga, M.B.; Araujo-Pereira, M.; Ladeia, A.M.; Andrade, B.B.; Lima, M.L. Lower Levels of Vitamin D Are Associated with an Increase in Insulin Resistance in Obese Brazilian Women. Nutrients 2021, 13, 2979. [CrossRef] [PubMed]

56. Zhao, H.; Zheng, C.; Zhang, M.; Chen, S. The relationship between vitamin D status and islet function in patients with type 2 diabetes mellitus. BMC Endocr. Disord. 2021, 21, 203. [CrossRef] [PubMed]

57. Engelman, C.D.; Meyers, K.J.; Iyengar, S.K.; Liu, Z.; Karki, C.K.; Igo, R.P., Jr.; Truitt, B.; Robinson, J.; Sarto, G.E.; Wallace, R.; et al. Vitamin D intake and season modify the effects of the GC and CYP2R1 genes on 25-hydroxyvitamin D concentrations. J. Nutr. 2013, 143, 17-26. [CrossRef] [PubMed]

58. Signorello, L.B.; Shi, J.; Cai, Q.; Zheng, W.; Williams, S.M.; Long, J.; Cohen, S.S.; Li, G.; Hollis, B.W.; Smith, J.R.; et al. Common variation in vitamin D pathway genes predicts circulating 25-hydroxyvitamin D Levels among African Americans. PLoS ONE 2011, 6, e28623. [CrossRef] [PubMed]

59. Su, M.; Chen, X.; Chen, Y.; Wang, C.; Li, S.; Ying, X.; Xiao, T.; Wang, N.; Jiang, Q.; Fu, C. UCP2 and UCP3 variants and gene-environment interaction associated with prediabetes and T2DM in a rural population: A case control study in China. BMC Med. Genet. 2018, 19, 43. [CrossRef] 